\title{
Interaction Techniques for Wall-Sized Screens
}

\author{
Lars Lischke \\ $\mathrm{HCI}$ Lab, VIS \\ University of Stuttgart \\ Stuttgart, Germany \\ lars.lischke@vis.uni-stuttgart.de albrecht.schmidt@vis.uni- \\ stuttgart.de \\ Jürgen Grüninger \\ Intel Visual Computing \\ Institute \\ Saarland University \\ Saarbrücken, Germany \\ juergen.grueninger@dfki.de \\ Khalil Klouche \\ Helsinki Institute for \\ Information Technology HIIT, Information Technology HIIT, \\ Department of Computer Department of Computer \\ Science, University of Helsinki, Science, University of Helsinki, \\ Finland \\ khalil.klouche@helsinki.fi \\ Finland \\ giulio.jacucci@helsinki.fi
}

\begin{abstract}
Large screen displays are part of many future visions, such as i-LAND [11] that describes the possible workspace of the future. Research showed that wall-sized screens provide clear benefits for data exploration, collaboration and organizing work in office environments. With the increase of computational power and falling display prices wall-sized screens currently make the step out of research labs and specific settings into office environments and private life. Today, there is no standard set of interaction techniques for interacting with wall-sized displays and it is even unclear if a single mode of input is suitable for all potential applications. In this workshop, we will bring together researchers from academia and industry who work on large screens. Together, we will survey current research directions, review promising interaction techniques, and identify the underlying fundamental research challenges.
\end{abstract}

\section{Author Keywords}

Large displays, wall-sized displays, public displays, interaction

\section{ACM Classification Keywords}

H.5.2 [User Interfaces]: Graphical user interfaces (GUI). 


\section{Background}

Marc Weiser's vision "Computing for the 21st century" [14] introduces three classes of devices to interact with digital content: "tabs", "pads" and "boards". "Tabs" and "pads" have already become commonplace with smartphones and tablet computers. In contrast, digital "boards" are still rarely used. However, there is a good chance that wall-sized display- "boards" will become commonplace within the next decade, like smartphones and tables did in the last decade. This development is in particular supported by software innovations. One example of software innovation is "Display as a Service" (DaaS) [7] which allows to display content from different sources across multiple single monitors without special synchronization hardware. By replacing specialized hardware with software like this, wall-sized displays and multi-display environments become significantly easier to set up.

Today, wall-sized displays are mainly used to visualize large and complex data. This is in particular beneficial, because humans are able to scan large areas quickly for objects and visual cues [5]. However, wall-sized public displays are becoming ubiquitous and are often used either for media installations or as information boards. Thereby, they do not only offer information to the user but can also help people to get in touch with each other [9].

In general, the success of wall-sized display installations depends highly on the interaction technique used in the particular setup. Already in 2006, Baudisch called for new user interfaces for wall-sized displays and tabletops [1]. In the future, wall-sized displays will not only be used in the context of professional visualizations and public displays, they will also become commonplace in office and some home environments. The large variety of interaction techniques and device form-factors we will use, need 1) a clear understanding of advantages and disadvantages of interaction techniques, and 2) an abstraction layer for software applications to map different interaction techniques in accordance to the specific given hardware setup of the user.

\section{Workshop Goals and Themes}

The goal of this workshop is to bring researchers together, who are focusing on interaction with wall-sized displays and multi-display environments, and to discuss challenges we are facing as research community. The results of the workshop will inspire work in the areas outlined below. As outcome of the workshop, we aim for a set of methods, challenges and topics in the field of wall-sized displays for possible joined publications and collaborations.

Appropriate input techniques depending on context of use We see multiple approaches to interact with wall-sized displays. Kuikkaniemi et al. [4] propose a system using direct touch for presentations in public settings. In contrast, Liu et al. [6] used a remote touch pad for data manipulation. Zadow et al. [13] proposed to use a touch display mounted to user's arm in addition to direct touch. Morris [8] argued for using gestures for interacting with large displays in private homes. Seyed et al. [10] designed a gesture set for interacting in multi-device environments. In such environments, wall-sized screens, tabletops and mobile devices are connected. The variety of areas of applications and interaction techniques indicate that there might not be one standard interaction technique for wall-sized displays. We need to design categories for areas of applications and interaction techniques. 
Content Representation on Wall-Sized Screens When using abundant display space, organizing visual content, such as application windows, becomes a challenging task. Grudin [2] discovered the issue of focal and peripheral awareness for multi-monitor users. We as humans are used to map locations to information and tools [3]. Additionally, large display space enables uses to switch between tasks without hiding other information. These findings indicate a high importance of arranging content on wall-sized displays. For wall-sized displays in office environments, we assume that there are different areas on screen for different tasks. The classical approaches used by common window management, are not sufficient for wall-sized displays. We need to design new concepts to assist users to arrange visual content.

\section{Novel Interaction Techniques}

There are multiple sensors, which seem to be underexplored as sensors to interact with wall-sized displays. On body sensors like EMG or brain computer interfaces $(\mathrm{BCl})$ promise to measure cognitive load. This technology could be used to adjust visual content on wall-sized display in accordance to the mental state of the users. This could lead to less information overload and thereby to a deeper engagement with the data set. In addition, $\mathrm{BCls}$ are becoming smaller and less pricey.

Turner et al. [12] proposes gaze in combination with touch to manipulate content on wall-sized displays. All these us to determine the user's mental state when interacting. Thereby the content can be adjusted to user's needs.

Towards standards for input techniques

The large number of different interaction techniques is challenging the design and implementation of successful applications. For example, there will be situations where, it might be interesting to use a wall-sized display without touch input, to give a presentation designed for a wall-sized display with touch input. To enable successful application development we need to design an abstraction model, which maps virtual actions to interaction techniques with specific input devices. There is also a need for mapping applications to a huge variety of screen sizes and screen resolutions. In an optimal case, this would not only cover different setups of wall-sized displays, but also desktop setups and mobile devices.

\section{Organizers}

Lars Lischke is a second year PhD-Student at the University of Stuttgart. Before he studied computer science in Stuttgart, Germany and Gothenburg, Sweden. In his $\mathrm{PhD}$ he focuses on new technologies for data exploration and interaction with large displays.

Jürgen Grüninger is a Researcher and PhD-Student at the Intel Visual Computing Institute and German Research Center for Artificial Intelligence (DFKI) in Saarbrücken.

Before, he studied computer science in Reutlingen, Germany. His research focuses on exchangeable interaction metaphors, devices and graphical interfaces and the combination of those for various application areas, including large displays.

Khalil Klouche is a PhD-Student at the Aalto School of Arts, Design and Architecture and a researcher at the Helsinki Institute for Information Technology (HIIT). He received a Master of Arts in Media Design at the Geneva University of Art and Design in 2013. His research focuses on designing interfaces supporting information exploration. Albrecht Schmidt holds the chair for $\mathrm{HCl}$ at the University of Stuttgart. He is co-founder of the ACM conference on Tangible and Embedded Interaction (TEI) and initiated the conference on Automotive User Interfaces. Albrecht is an associate editor in chief of the IEEE Pervasive Computing Magazine and edits a column 
on invisible Computing in the IEEE Computer Magazine. Philipp Slusallek is Scientific Director at the DFKI, where he heads the research area Agents and Simulated Reality. He is also Director for Research at the Intel Visual Computing Institute. His research interests are focused on novel service-oriented architectures for 3D-Internet technology, integrating research in areas such as real-time realistic graphics, artificial intelligence, high-performance computing as well as security by design for creating distributed, immersive, collaborative environments for simulation, analysis, visualization, and training.

Giulio Jacucci is Professor at the Department of Computer Science and director of the Network Society Programme at the HIIT. His research field is in $\mathrm{HCl}$ including: mobile interaction, mediated social interaction, multimodal and implicit interaction, haptics and tangible computing, mixed reality, and persuasive technologies. He co-authored Design Things by MIT press. He is also co-founder and member of the board of directors of MultiTaction.com. MultiTouch Ltd. is a spin-off from a EU FET and leader in modular interactive screens.

Acknowledgment: This work is partially funded by the European Community's H2020 Program under the funding scheme "FETPROACT-1-2014: Global Systems Science (GSS)", grant agreement \# 641191 CIMPLEX.

\section{References}

[1] Baudisch, P. Interacting with Large Displays. Computer 39, 4 (2006), 96-97.

[2] Grudin, J. Partitioning digital worlds. In Proc. CHI (2001), 458-465.

[3] Kirsh, D. The intelligent use of space. Artificial Intelligence 73, 1-2 (1995), 31-68.

[4] Kuikkaniemi, K., Vilkki, M., Ojala, J., Nelimarkka, M., and Jacucci, G. Introducing Kupla UI : A Generic Interactive Wall User Interface Based on
Spherical Widgets. In Proc. ITS (2013), 301-304.

[5] Lischke, L., Mayer, S., Wolf, K., Henze, N., Schmidt, A., Leifert, S., and Reiterer, H. Using Space: Effect of Display Size on Users' Search Performance. In Proc. CHI EA (2015), 1845-1850.

[6] Liu, C., Chapuis, O., Beaudouin-Lafon, M., Lecolinet, E., and Mackay, W. E. Effects of display size and navigation type on a classification task. In Proc. CHI (2014), 4147-4156.

[7] Löffler, A., Pica, L., Hoffmann, H., and Slusallek, P. Networked Displays for VR Applications: Display as a Service. In ICAT/EGVE/EuroVR (2012), 37-44.

[8] Morris, M. R. Web on the wall. In Proc. ITS, ACM Press (2012), 95.

[9] Peltonen, P., Kurvinen, E., Salovaara, A., Jacucci, G., Ilmonen, T., Evans, J., Oulasvirta, A., and Saarikko, P. It's Mine, Don't Touch! In Proc. CHI (2008), 1285.

[10] Seyed, T., Burns, C., Costa Sousa, M., Maurer, F., and Tang, A. Eliciting usable gestures for multi-display environments. In Proc. ITS (2012), 41.

[11] Streitz, N. A., Geiß ler, J., Holmer, T., Konomi, S., Müller-Tomfelde, C., Reischl, W., Rexroth, P., Seitz, P., and Steinmetz, R. i-LAND: an interactive landscape for creativity and innovation. In Proc. CHI (1999), 120-127.

[12] Turner, J., Alexander, J., Bulling, A., and Gellersen, H. Gaze+RST: Integrating Gaze and Multitouch for Remote Rotate-Scale-Translate Tasks. In Proc. CHI (2015), 4179-4188.

[13] von Zadow, U., Büschel, W., Langner, R., and Dachselt, R. SleeD: Using a Sleeve Display to Interact with Touch-sensitive Display Walls. In Proc. ITS (2014), 129-138.

[14] Weiser, M. The Computer for the 21st Century Scientific American 265, 3 (1991), 94-104. 